\title{
Utilização do ciclo PDCA para melhoria de qualidade e aumento de produtividade em uma multinacional do polo industrial de Manaus
}

\author{
Use of the PDCA cycle to improve quality and increase productivity in a multinational in the
}

Manaus industrial pole

Uso del ciclo PDCA para mejorar la calidad y aumentar la productividad en una multinacional en el polo industrial de Manaus

Recebido: 09/10/2021 | Revisado: 16/10/2021 | Aceito: 19/10/2021 | Publicado: 20/10/2021

Francisco do Espirito Santo Rodrigues Ferreira ORCID: https://orcid.org/0000-0002-1414-2929 Universidade Federal do Pará, Brasil E-mail: eng.francisco@live.com

Edilson Marques Magalhães ORCID: https://orcid.org/0000-0003-3056-0757 Universidade Federal do Pará, Brasil E-mail: magalhaes@ufpa.br

\begin{abstract}
Resumo
$\mathrm{Na}$ empresa multinacional em questão, foi observado que os indicadores de qualidade e produtividade não estavam sendo alcançados. O presente trabalho tem como objetivo a aplicação da ferramenta PDCA nas linhas de montagens da companhia em estudo para se alcançar as metas relacionadas aos indicadores de produtividade e qualidade. A metodologia aplicada para se alcançar os objetivos foi desenvolvida a partir de pesquisa bibliográfica, verificação da situação atual de todo o processo, onde foi realizado todo o levantamento dos principais problemas e suas causas. Em relação aos fatores que causam não conformidades dos indicadores, verificou-se a necessidade de criação de uma sistemática para desenvolvimento das atividades com envolvimentos de vários setores para aprimorar a comunicação e uma frequência de verificação diária dos andamentos de cada projeto. Como resultado da implantação da sistemática e principalmente a utilização da ferramenta PDCA, houve uma redução nas perdas de qualidade em aproximadamente $0,01 \%$ e aumento de produtividade em $0,16 \%$ nos primeiros seis meses.
\end{abstract}

Palavras-chave: Qualidade; Produtividade; PDCA; Sistemática.

\begin{abstract}
In the multinational company in question it was realized that the quality and productivity indicators were not being achieved. The present work has as purpose the application of the PDCA tool in the assembly lines of the company under study to reach the goals related to the productivity and quality indicators. The methodology applied to achieve the goals was developed based on bibliographical research, checking the current situation of the whole process and where the entire survey of the main problems and their causes was carried out. About to the factors that cause non-conformities in the indicators it was necessary to create a systematic for the development of activities involving various departments to improve communication and a frequency of daily checking of the progress of each project. As a result of the implementation of the systematic and mainly the use of the PDCA tool, there was a reduction in quality losses of approximately $0.01 \%$ and an increase in productivity of $0.16 \%$ in the first six months.
\end{abstract}

Keywords: Quality; Productivity; PDCA; Systematic.

\section{Resumen}

En la empresa multinacional en cuestión se percató que no se estaban cumpliendo los indicadores de calidad y productividad. El presente trabajo tiene como finalidad la aplicación de la herramienta PDCA en las líneas de montaje de la empresa en estudio para alcanzar las metas relacionadas con los indicadores de productividad y calidad. La metodología aplicada para la consecución de los objetivos se desarrolló a partir de la investigación bibliográfica, comprobando la situación actual de todo el proceso y donde se realizó el relevamiento completo de los principales problemas y sus causas. Sobre los factores que causan no conformidades en los indicadores fue necesario crear una sistemática para el desarrollo de actividades que involucren a varios departamentos para mejorar la comunicación y una frecuencia de control diario del avance de cada proyecto. Como resultado de la implementación sistemática y principalmente del uso de la herramienta PDCA, hubo una reducción en las pérdidas de calidad de aproximadamente $0.01 \%$ y un aumento en la productividad de $0.16 \%$ en los primeros seis meses.

Palabras clave: Calidad; Productividad; PDCA; Sistemática. 


\section{Introdução}

O atual cenário social e principalmente econômico não apenas do Brasil, mais do mundo, é de incertezas. Isso faz com que as organizações não tenham margens para desperdícios e retrabalhos no desenvolvimento de suas atividades, pois estas anormalidades geram custos adicionais reduzindo a competitividade em um mercado cada vez mais exigente e competitivo.

Desta forma, faz-se necessário que as empresas repensem os seus procedimentos e técnicas para alcançar os seus resultados conforme o planejado, priorizando sempre pela qualidade. O termo "qualidade" pode ser considerado uma palavra antiga e bastante popular. Durante um bom período as pessoas traziam na mente um conceito objetivo e claro sobre a palavra qualidade, esse conceito tem sido usado desde meados do século IXX e início do século XX, onde envolve uma agregação de técnicas com ênfase na melhoria contínua, atendendo dessa forma às exigências dos clientes e diminuindo o retrabalho (Gerolamo et. al., 2014).

Nesse contexto de melhoria continua e gestão de qualidade, existem métodos e ferramentas que possibilitam a identificação, coordenação e a execução das melhorias dos processos de produtos ou serviços. Dentre esses métodos na ênfase da melhoria continua o ciclo PDCA é um dos mais utilizados nos processos produtivos. A sigla PDCA em português, significa planejar, executar, verificar e agir. O método PDCA consegue conduzir de maneira sistêmica o encontro da solução do problema na gestão de qualidade em cooperação com outros tipos de ferramentas, (Marshall et al. 2010). A utilização do ciclo PDCA possibilita a redução dos custos da empresa, melhorando assim o seu processo produtivo. (Duppre et al., 2015).

A motivação pela qual se justifica este estudo sobre a aplicação da metodologia PDCA em uma organização industrial é evitar que ocorra resultados não satisfatórios devido ao não alcance das metas conforme o planejado referente aos indicadores de qualidade e produtividade ou taxa de ocupação. Portanto, este trabalho irá mostrar o passo a passo da aplicação do ciclo PDCA, dando-se ênfase, principalmente, na etapa onde ocorre os maiores problemas, que é a etapa do planejamento e com isso espera-se alcançar as metas referente a qualidade e produtividade com o mínimo de diferença possível entre o resultado esperado e o alcançado.

\section{Revisão da Literatura}

No desenvolvimento desta revisão de literatura serão abordados tópicos como: O conceito de PDCA, a evolução histórica da ferramenta e a definição de cada fase da metodologia, tendo um enfoque maior para a fase PLAN (P), pois esta fase consideramos que além de ser o início, ela norteia todos as fases seguintes que são EXECUTAR (D), CHECK (C) e AGIR (A), portanto podemos considerar como sendo a mais importante do ciclo PDCA. Estas fases da metodologia serão apresentadas no decorrer deste trabalho.

\subsection{Conceito de PDCA}

É uma ferramenta de melhoria na gestão que gera oportunidades de melhorias, possibilitando que as diretrizes traçadas pelo planejamento estratégico sejam viabilizadas, no entanto é de extrema importância o engajamento de todos os colaboradores da organização com o método. Este ciclo é ininterrupto e visa a melhoria contínua, pois, usando o que foi aprendido em uma aplicação do ciclo PDCA, pode-se começar outro ciclo, em uma tentativa mais complexa e, assim, sucessivamente. Com isso, o último ponto sobre o ciclo PDCA se torna o mais importante, pois este assumirá um novo começo (Falconi, 2017).

Kitahara (2016), afirma que o ciclo PDCA, também chamado de ciclo da administração, é uma forma de fazer avançar o trabalho. Como parte do processo de manutenção e melhoria da situação atual, a primeira etapa é definir um plano (PLAN). em seguida, implemente o plano (DO) e verifique os resultados (CHECK). Ações corretivas são tomadas conforme necessário (ACTION), o que gera um novo plano no próximo ciclo. 
Seguindo esta linha de raciocínio, podemos afirmar o conceito de PDCA está diretamente ligado ao conceito de melhoria continua, a ferramenta deve ser utilizada como um ciclo dinâmico, ou seja, a conclusão de um ciclo sempre irá terminar no início do próximo ciclo, conforme ilustra Figura 1.

Figura 1 - Ciclo PDCA.

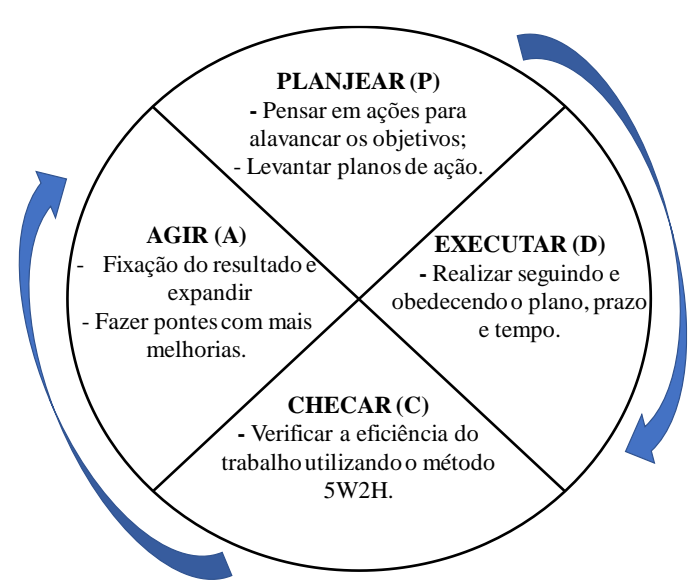

Fonte: Apostila de Treinamento da Empresa em Estudo.

\subsection{Evolução do PDCA ao longo dos anos}

A definição de métodos e melhorias, que atualmente é conhecido pela sigla PDCA, teve seu início na década de trinta. Em 1931, Shewart publica o livro Economic Control of Quality of Manufactured Product, o qual confere um caráter cientifico as questões relacionadas a Qualidade (Souza, 1997).

Moen e Norman (2006) afirmam que o ciclo PDCA teve a sua origem com a palestra de William Edwards Deming, no Japão, em 1950, onde ele promoveu melhorias no ciclo, dando origem a sua forma mais conhecida, conforme apresentado na Figura 2, a qual foi intitulada de ciclo de Deming.

Figura 2 - Ciclo de DEMING (1950).



Fonte: Adaptado de Moen e Norman (2006).

A relação do ciclo de Deming da década 1950 com o atual, poderemos ver a seguir conforme abaixo:

- Projetar: É a primeira etapa e exige a necessidade de pensar, arquitetar um determinado objetivo, logo faz referência diretamente ao PLAN (P);

- Produzir: É a materialização do objetivo, ou seja, é a colocação em prática do primeiro passo, portanto faz referência ao Executar (D);

- Vender: Neste caso seria o retorno da materialização, o resultado do objetivo, ou seja, a confirmação, portanto faz referência ao Check $(\mathrm{C})$; 
- Redesenhar: É a correção do projeto, aperfeiçoamento dos pontos fortes e eliminação dos pontos fracos, ou seja, faz referência a tomada de Ação (A).

Em consonância com estas alterações ao longo dos anos e fortalecendo o seu significado e aplicabilidade, (Moen e Norman 2006), assim como a Fundação Calouste Gulbenkian (2014), relatam as alterações ocorridas e em 1996, o modelo foi renomeado para "Modelo de Melhoria" e foram acrescentadas três perguntas ao modelo existente, o qual foi reforçado novamente em 2009 por diversos investigadores. Desta forma, o modelo é o que consta na Figura 3.

Figura 3 - Modelo de Melhoria, 1996, 2009.

\section{Modelo de Melhoria}

O que estamos tentando realizar?



Fonte: Moen e Norman (2006).

\subsection{As fases da metodologia PDCA}

\subsubsection{Fase do planejamento $(P)$}

Kitahara (2016), define esta fase como primordial para o desenvolvimento e o êxito da utilização do ciclo PDCA. Para isso devemos fazer uma subdivisão, conforme a seguir:
a) Localização do problema;
b) Entender a situação atual;
c) Estabelecer objetivos;
d) Analisar o problema;
e) Preparar o plano de ação.

\subsubsection{Localização do problema}

Identificar a anormalidade é o primeiro estágio da solução do problema. Mais especificamente, significa perceber que existe um desvio do estado ideal (lacuna entre a situação atual e a situação ideal). Na Tabela 1 abaixo sugere perspectivas potenciais a partir das quais o problema pode ser identificado. 
Tabela 1 - Perspectiva potenciais para identificar problemas.

\begin{tabular}{|c|c|c|}
\hline Políticas / Procedimentos & Ambiente de trabalho & Foco futuro \\
\hline $\begin{array}{l}\text { - A política / procedimento } \\
\text { existente inibe a conclusão } \\
\text { eficiente do trabalho; } \\
\text { - A política / procedimento } \\
\text { existente impacta negativamente a } \\
\text { moral do funcionário; } \\
\text { - Nenhuma política ou } \\
\text { procedimento existe para garantir } \\
\text { a atividade de trabalho consistente }\end{array}$ & $\begin{array}{c}\text { - Os problemas que o cercam são } \\
\text { persistentemente incômodos ou } \\
\text { incompatíveis; } \\
\text { - O layout do equipamento / } \\
\text { escritório inibe a conclusão } \\
\text { eficiente do trabalho } \\
\text { - Pessoas em seções relacionadas } \\
\text { ou na fase anterior e / ou na } \\
\text { próxima fase expressam } \\
\text { insatisfação com a produção / } \\
\text { resultados. }\end{array}$ & $\begin{array}{l}\text { - Assuntos de estado futuro ou } \\
\text { problemas antecipados requerem a } \\
\text { resolução de problemas; } \\
\text { - Mesmo na ausência de } \\
\text { insatisfação atual, inicie a } \\
\text { resolução de problemas em um } \\
\text { esforço para elevar o trabalho a } \\
\text { um nível mais alto; } \\
\text { - Coloque a política geral de } \\
\text { gestão em termos simples e } \\
\text { identifique o que deve ser feito } \\
\text { para alcançá-los. }\end{array}$ \\
\hline
\end{tabular}

Fonte: Kitahara (2016).

\subsubsection{Entender a situação atual}

Kitahara (2016), afirma que compreender a situação atual significa inspecionar como o problema ocorreu e priorizar a área problemática. Será possível notar que não há necessariamente uma única área problemática. É por isso que, depois de entender a situação atual, podemos nos concentrar em um alvo prioritário em busca de uma solução.

\subsubsection{Estabelecer objetivos}

Iwahana (2013), afirma que o tipo de objetivos que estabelecemos deve ser desafiador, evocando a sensação de que é exatamente porque esse obstáculo é tão difícil que quero superá-lo. Quando as pessoas sentem que um problema ou a ponte é sua responsabilidade, estabelecer uma meta mais elevada tenderá a aumentar o conhecimento técnico dos envolvidos.

\subsubsection{Analisar o problema}

Esta é a etapa onde se deve fazer todas as análises possíveis para que se tenha o máximo de entendimento da anormalidade. A capacidade de identificar a natureza de um problema não é algo fácil. Praticar verificação no local, perguntar repetidamente "por quê?", Fazer esforços para entender a verdadeira causa, esses são os tipos de processos pelos quais essa habilidade pode ser cultivada (Iwahana, 2016).

\subsubsection{Preparar o plano de ação}

Conforme Campos (1996), “os planos de ação colocam o gerenciamento em movimento”. Dessa forma a metodologia viabiliza a ação concreta no gerenciamento, delegando responsabilidade aos envolvidos no palno de ação. Para facilitar a obtenção de exito, a ferramenta mais indica é $5 \mathrm{~W} 1 \mathrm{H}$, onde a mesma consiste elaborar um plano de ação levando em consideração seis perguntas, conforme abaixo:

- WHAT (O QUE) - Define o que será executado, contendo explicação da ação a ser tomada;

- WHEN (QUANDO) - Define quando será executado a ação;

- WHO (QUEM) - Define quem é o responsavel pela ação;

- WHERE (ONDE) - Define onde será executada a ação;

- WHY (POR QUE) - Define as razões para a tomada de ação;

- HOW (COMO) - Define o detalhamento de com será tomada a ação. 


\subsubsection{Fase da execução (D)}

Segundo Kitahara (2016), quando se trata de implementar contra-medidas acordadas, é importante que a atitude básica seja a disposição para ser intransigente e persistente até um final bem-sucedido. Quando você entra no estágio de implementação, pode haver problemas e obstáculos ininterruptos.

\subsubsection{Fase check / study (C)}

Ao realizar esta etapa será possível refletir sobre qualquer caso em que os resultados não tenham saído de acordo com as expectativas, mas mesmo quando os resultados corresponderem às expectativas, será possível rever o caminho a cada etapa. progrediu para considerar o que era particularmente bom e para utilizar essa reflexão para levar o trabalho a um nível superior. Fazer isso é o primeiro passo para a construção de metas ou medidas ainda mais elevadas (Kitahara, 2016).

\subsubsection{Fase agir (A)}

Segundo Iwahana (2013), olhando para a contramedida que produziu os resultados, o processo de definição dos meios e dispositivos de manutenção e administração e de estabelecimento ou revisão de normas e manuais tem por objetivo a padronização em nível organizacional. Esse tipo de implementação cruzada está vinculado ao avanço eficiente da solução de problemas em toda a organização.

\subsection{Aplicações do ciclo PDCA}

Rodrigues (2017) utilizou o ciclo PDCA para aumentar a produtividade em atividades de manutenção de Shuts de correias transportadoras em uma indústria de mineração. Após a implementação, a metodologia teve como grande contribuição a identificação e consolidação de melhorias no sistema de manutenção e consequentemente o aumento na confiabilidade e disponibilidade dos equipamentos.

Suski (2020) empregou o ciclo PDCA em um processo de usinagem de uma empresa do ramo metal mecânico e teve como objetivo a redução de custo de insertos. Os resultados mostraram a melhoria na vida útil das ferramentas de usinagem e, consequentemente, redução do custo dos insertos por tonelada de peças usinadas.

Sousa (2020) aplicou o ciclo PDCA para a redução de custos e perdas em uma distribuidora de hortifrúti. Após implementação das melhorias teve como resultado positivo a redução em aproximadamente $50 \%$ o índice de devoluções e consequentemente a redução de custo.

De Paula (2021) empregou o ciclo PDCA na logística reversa de uma indústria do ramo alimentício objetivando gerar soluções práticas e viáveis para o problema de insatisfação dos principais clientes da organização e teve como principais benefícios um aumento no controle e gestão de toda a cadeia produtiva do processo.

Pereira (2021) utilizou a metodologia PDCA no processo logístico e no armazenamento do hortifrúti tomate em uma empresa de transporte fluvial e teve como objetivo investigar as principais causas que afetam a qualidade do hortifrúti.

\section{Estudo de caso: Utilização do ciclo PDCA para melhoria de qualidade e aumento de produtividade}

\subsection{Cenário atual da empresa}

Este trabalho baseou-se em análise de informações de perdas de qualidade e taxa de ocupação ou produtividade nas linhas de montagem de uma multinacional do Polo Industrial de Manaus (PIM) nos anos de 2019 e 2020. Todas essas informações obtidas eram armazenadas em planilhas onde alguns setores e pessoas envolvidas na atividade tinham acessos às informações e este acompanhamento foi feito de forma diária em um período de 18 meses. A empresa em estudo produz peças para motos e carros, o seu parque industrial de máquinas é de aproximadamente trezentas e tem um total de vinte linhas de montagem, porém 
este estudo se concentra apenas no setor Y que produz peças para carros e este setor tem cinco linhas de produção. Todas as linhas do setor fizeram parte deste estudo.

\subsection{Universo e amostra}

Devido a política de confidencialidade e por questões de segurança da informação, alguns dados tipo origem e nome da empresa, nome de processos e produtos, não poderão ser mencionados neste trabalho, no entanto, será mostrada toda a lógica desenvolvida utilizando a metodologia PDCA para se alcançar os objetivos ou metas definidas.

\subsection{Análise de dados}

Nesta etapa foi feita a organização de todos os dados, principalmente dos resultados alcançados neste trabalho e além disso tem como objetivo quantificar e evidenciar os ganhos obtidos com a melhoria implantada. Para facilitar o entendimento, os resultados serão mostrados através de representação gráfica (Histograma) onde se procurou mostrar um comparativo da situação antes e após implantação da melhoria que que é a utilização da metodologia PDCA.

\subsection{Metodologia}

É de extrema importância saber o método a ser adotado para se ter um melhor desempenho no desenvolvimento da atividade e além disso, um entendimento de forma clara e de fácil compreensão. Portanto faz-se necessário ter o conhecimento da situação atual, onde se deseja chegar e os meios para se alcançar os objetivos, desta forma facilitando o êxito do trabalho. De acordo com Pereira (2018) a metodologia para desenvolvimento de um estudo de caso pode ser por métodos qualitativos e quantitativos.

Para Estrela (2018) um estudo de qualidade precisa estar bem delineado e o objetivo do trabalho define a metodologia a ser adotada e normalmente em estudos de casos são adotados os métodos básicos que são qualitativos e quantitativos.

\subsubsection{Métodos qualitativos}

Pereira (2018) descreve que este tipo de metodologia requer uma interpretação de dados e normalmente as fontes são através pesquisas e entrevistas, onde os resultados são apresentados de forma descritiva. Na maioria das vezes esta metodologia pode se transformar em quantitativa.

Estrela (2018) afirma que este método é utilizado para avaliar comportamentos, ideias e situações em que não estejam apresentadas de forma clara, sendo necessário um estudo mais aprofundado sobre determinada situação ou fenômeno especifico.

\subsubsection{Métodos quantitativos}

Normalmente nesta metodologia utiliza-se cálculos numéricos. Pereira (2018) define que as coletas de dados neste método são através de medições e os resultados são analisados través de fórmulas matemáticas, cálculos estatísticos, probabilidades e outros métodos numéricos. Este método pode ser aplicado em diversas áreas, desde a saúde até a engenharia

Segundo Estrela (2018), esta metodologia é normalmente aplicada quando se tem uma situação ou objetivo previamente definido e através deste é método é possível fazer comparações em situações diferentes utilizando com predominância dados numéricos.

\subsubsection{Método adotado}

De acordo com os autores citados acima neste trabalho, podemos caracterizar este estudo em questão com predominância de método quantitativo, uma vez que os objetivos estão definidos, as fontes de dados são através de medições e 
para análise dos resultados foi utilizado dados de porcentagem, onde foi feito um comparativo entre duas situações. Yin (2015) salienta que os métodos qualitativos e quantitativos não se excluem e sim eles se complementam, desta forma é possível ter um melhor resultado dos fenômenos em estudos com ambas metodologias.

Com objetivo de organizar e ter um melhor entendimento da metodologia adotada neste trabalho, a pesquisa foi dividida nas seguintes etapas do fluxograma, conforme mostrado na Figura 4.

Figura 4 - Fluxograma das etapas do ciclo PDCA.

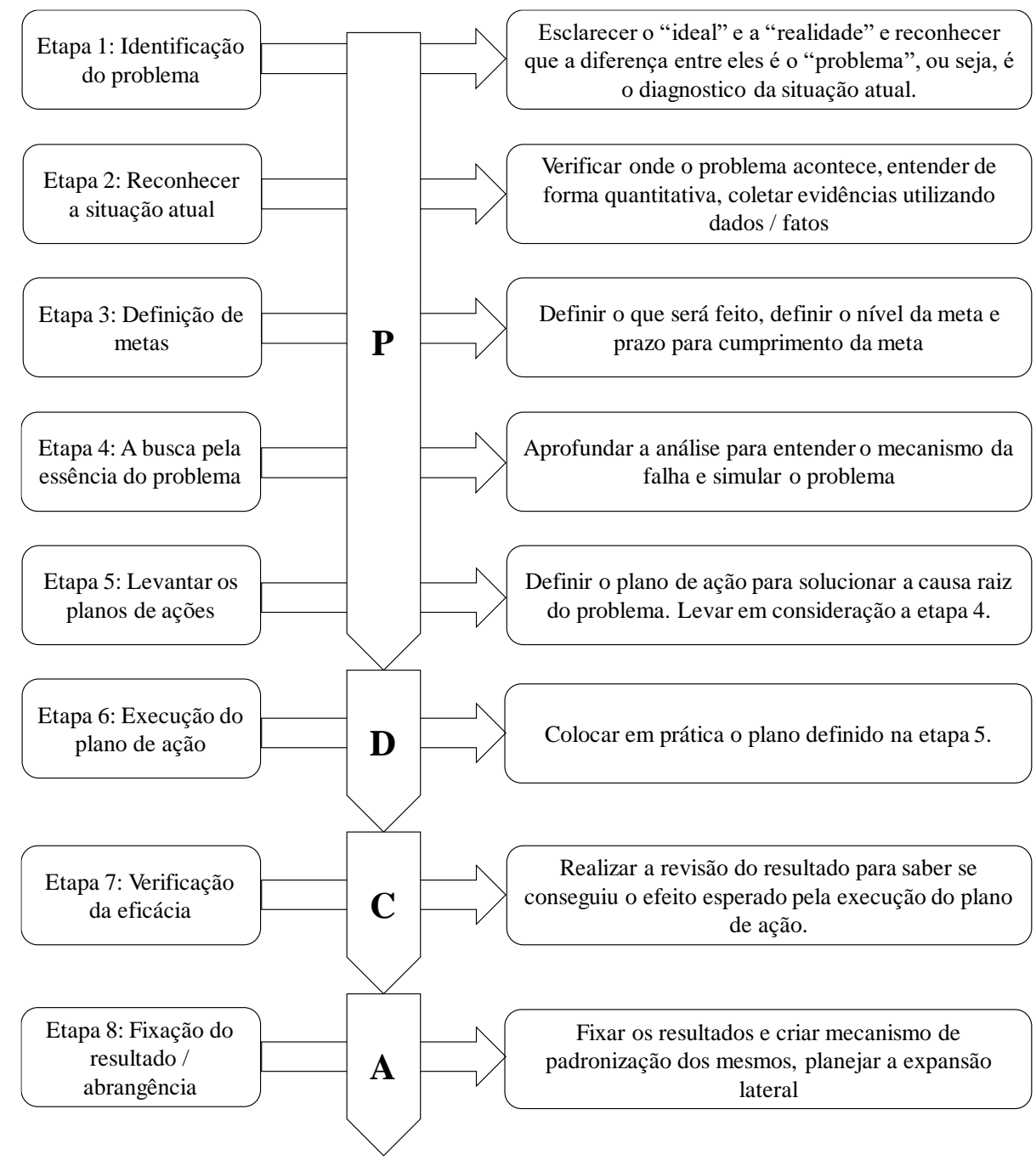

Fonte: Autores.

\subsubsection{Etapa 1- Identificação do problema}

Nesta etapa será feito um diagnóstico da situação atual, fazendo um levantamento de todo o cenário, mostrando as principais anormalidades encontradas e com essas informações, ter conhecimento o quanto distante está da situação ideal. A lógica utilizada para se fazer as análises foram verificação onde o problema ocorre e de que forma acontece os mecanismos para gerar as anormalidades, quantificação dos problemas, tabela de priorização e principalmente a utilização de gráficos para um melhor entendimento do cenário.

\subsubsection{Etapa 2- Reconhecer a situação atual}

Nesta etapa é de extrema importância a definição de que forma irá ser feita a visualização do problema, esta definição deve ser feita de forma que o entendimento da anormalidade não seja de forma superficial, por isso é importante fazer a 
verificação onde o problema está acorrendo, ter evidências, coletar dados, fazer a quantificação e analisar o impacto de cada anormalidade para posterior priorização na tomada de ações.

\subsubsection{Etapa 3- Definição das metas}

Quando definimos as metas, estamos informando até que ponto será solucionado um problema e normalmente a definição é feita baseada em "o que (tópicos)", "até que nível" e até quando (prazo da meta)".

\subsubsection{Etapa 4- A busca pela essência do problema}

Para que seja possível alcançar o objetivo definido, é preciso identificar a verdadeira causa da anormalidade através de análise dos mecanismos que geram o problema. É preciso aprofundar os conhecimentos referente ao problema e não se limitar em conceitos conhecidos ou experiência / resultados passados, levantar todas possíveis causas e realizar abrangência para outros produtos e processos ou sistema de gestão.

Em casos de produtos, é preciso reproduzir o problema e se o resultado da reprodução for igual a anormalidade, é dada continuidade para o próximo passo, porém se o resultado for diferente é preciso fazer novamente a análise.

Para processo ou sistema de gestão é necessário fazer uma reflexão do passado e das atividades anteriores, evidenciando quais atividades estavam sendo feitas e os envolvidos neste cenário. O resultado dessa análise resulta como mecanismo de falha problemas estruturais, tais como: Gerenciamento, Sistemática e Falta de conhecimento.

\subsubsection{Etapa 5- Levantar o plano de ação}

Nesta etapa o objetivo é organizar as informações e em seguida definir o melhor plano e verificar se não há necessidade de fazer abrangências para outros produtos e processos ou sistema de gestão. O plano deve ter informação de: $\mathrm{O}$ que, prazo, local, responsável, qual é método ou ferramenta será utilizada e o custo para aplicação.

\subsubsection{Etapa 6- Execução do plano de ação}

Esta etapa coloca em prática todo resultado do trabalho dos cinco passos anteriores. Alguns pontos de atenção devemos ter para executar os planos de ação como: Compartilhar a meta ou a condição que queira concretizar o plano de ação, identificar as suas responsabilidades ao compartilhar o plano de execução, ter comunicação de tempo em tempo para compartilhar e transmitir informações adequadas aos superiores ou à equipe e criar uma forma visual para que todos possam ver o andamento do plano de execução.

\subsubsection{Etapa 7- Verificação da eficácia}

Esta etapa é onde ocorre a "revisão do resultado" para saber se obteve o efeito esperado pela execução do plano de ação e também serve para ter conhecimento se o andamento da execução foi adequado.

\subsubsection{Etapa 8- Fixação do resultado / Abrangência}

Nesta etapa devem ser apresentados todo o resultado da implementação das etapas anteriores e além disso, tem como objetivo criar um sistema de manutenção de resultado e que se consiga padronizar e também verificar a possibilidade de expandir os resultados para outros processos onde a lógica possa ser aplicada. 


\section{Resultados e Discussão}

Neste capítulo, em resultado da metodologia definida no capítulo anterior, serão analisados os benefícios obtidos em cada etapa do ciclo do PDCA após utilização da ferramenta. O estudo teve início em janeiro de 2020 e isso se deu devido ao não atingimento das metas planejadas pela companhia. Durante um mês foram feitas reuniões diárias para entender o cenário já apresentado no capítulo anterior e em seguida durante três meses foram feitos estudos entre vários setores da empresa para o desenvolvimento e aplicação da metodologia do ciclo PDCA. A seguir será apresentado os benefícios obtidos em cada etapa.

\subsection{Benefícios da etapa 1}

Esta etapa serviu como o alicerce para o desenvolvimento do trabalho, foi onde identificamos que tínhamos anormalidades e além disso, nos mostrou a importância e o tamanho do problema através da identificação de forma clara a diferença entre o ideal e a realidade, conforme mostrado na Figura 5. A fonte de dados para esta identificação foi principalmente através de relatórios gráficos.

Figura 5 - Identificação do problema.

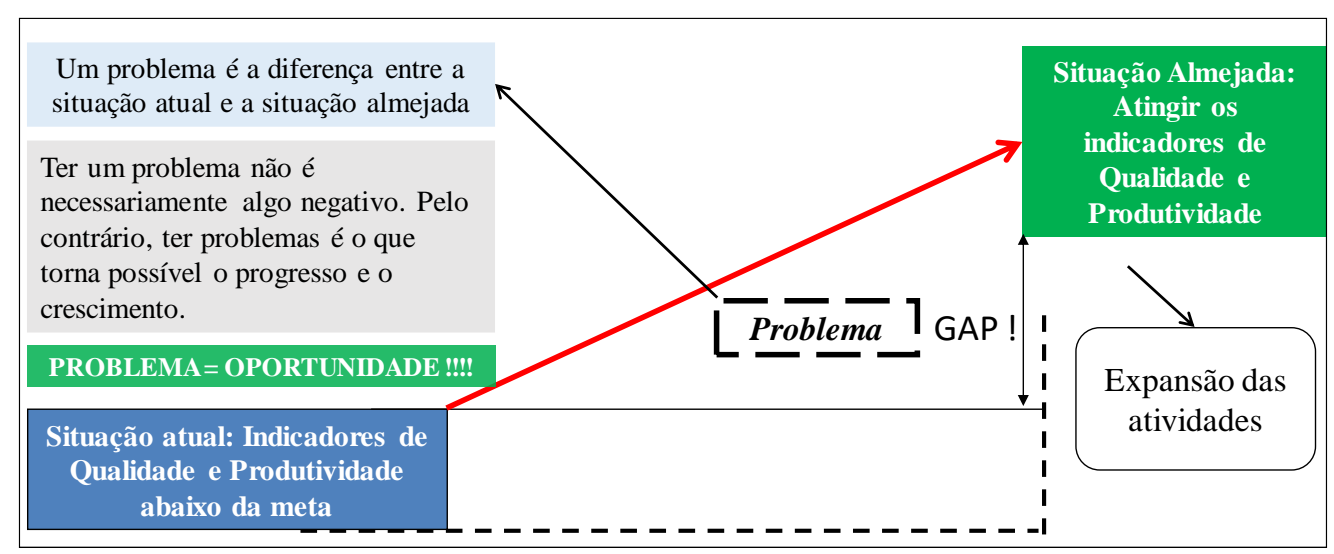

Fonte: Autores.

Para facilitar o entendimento e até mesmo possíveis informações faltantes, foi utilizado figuras ou gráficos com informações que direcionem a visualização do problema de forma correta. Após entender o problema quantitativamente, é preciso fazer a estratificação do mesmo e em seguida qual devemos priorizar para tomar e solucionar a anormalidade. Para priorizar uma atividade são levados em consideração alguns pontos como: impacto no resultado e / ou dificuldade na execução.

Para a organização em que o estudo foi realizado, a lógica de definição de metas é sempre no início do ano fiscal, onde a companhia faz a sua política de meio ambiente, produtividade, segurança e qualidade e de acordo essas informações os setores elaboram a sua política departamental e cronograma de atividades para atingir as metas do setor e consequentemente da empresa. Em relação meta do indicador de perdas de qualidade a definição foi a redução de 0,01\% em relação ao ano de 2019, enquanto que o indicador de taxa de ocupação foi uma redução de 0,16\% em relação ao ano de 2019. Essas foram metas definidas e esperadas pela a organização para o ano de 2020. Após entendimento do problema, da forma de visualização do mesmo e o nível de cada meta, foi importante aprofundar os mecanismos das falhas, ou seja, entender de que forma aconteciam para evitar possíveis reincidências.

\subsection{Benefícios da etapa 2}

Esta etapa nos ensinou justamente como lidar em uma situação ou cenário onde existia vários gargalos e que certamente sem a organização de informações dos dados, a forma correta de coletar, a forma de visualizar, a quantificação e até mesmo a 
priorização não seriam possíveis alcançar os objetivos inicialmente planejados. O cenário no ano de 2019 para as empresas de manufatura começou muito promissor no que diz a respeito ao volume de vendas mensal. O cenário político e econômico favorecia as expectativas positivas e nos primeiros meses isso se confirmou. A cada mês que se passava a demanda mensal aumentava e o ambiente na organização aparentava estar cada dia melhor, ninguém esperava que um cenário totalmente positivo se tornaria totalmente adverso em dezembro de 2020 não apenas nacionalmente, porém mundialmente em virtude de uma pandemia que assola o mundo até os dias atuais, e com isso automaticamente houve queda de demanda no volume de vendas. Todo este cenário descrito acima é possível verificar na Figura 6 que representa a demanda mensal em 2019 e onde mostra s seguintes informações: Demanda planejada, demanda real e a diferença entre demanda real x planejada.

Figura 6 - Demanda mensal em 2019.

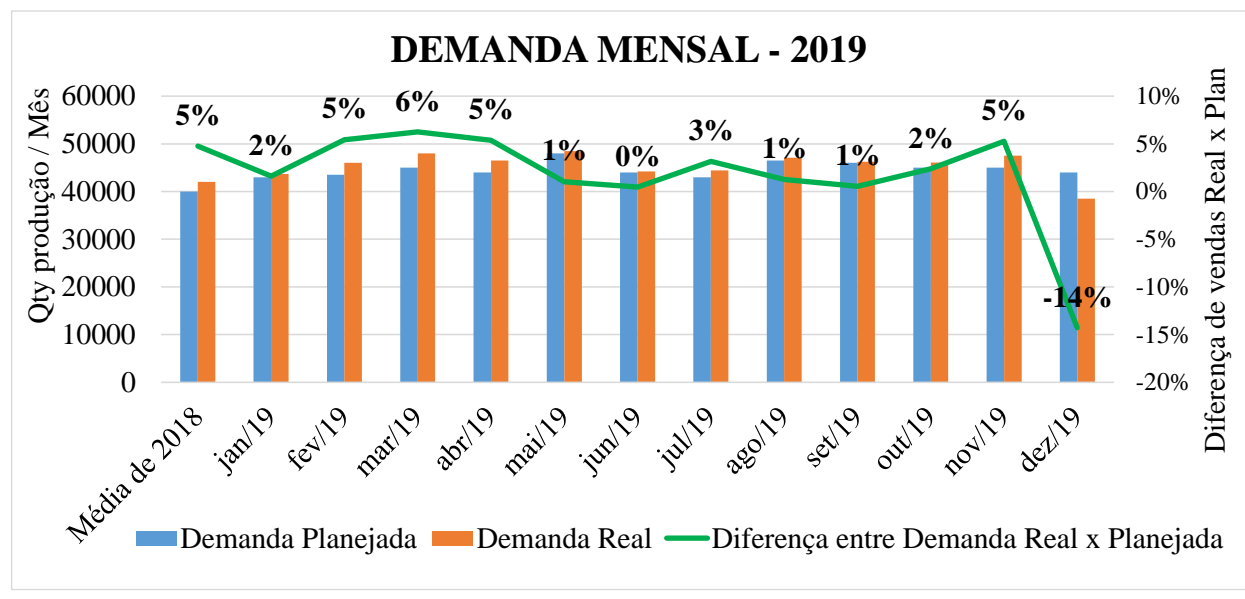

Fonte: Autores.

Na Figura acima, é possível observar que em apenas um mês a demanda real ficou abaixo da demanda planejada. Este cenário em que a demanda real sempre fica acima da demanda planejada tem os seus deméritos, pois algumas atividades de melhorias acabam não sendo feitas ou implantadas, em virtude de o ambiente não ser propicio, já que a organização estar vendendo mais que o planejado e certamente a verificação do andamento das atividades não são feitos corretamente, as cobranças diminuem e consequentemente não ocorrem melhorias. Todo esse relato ocorreu com esta organização, onde no início do ano fiscal de 2019 foram planejadas atividades de melhoria de qualidade e melhoria de produtividade, porém conforme mostra as Figuras 7 e 8 que representam perdas de qualidade e taxa de ocupação respectivamente, não foram implantadas.

Figura 7 - Perdas de qualidade em 2019.

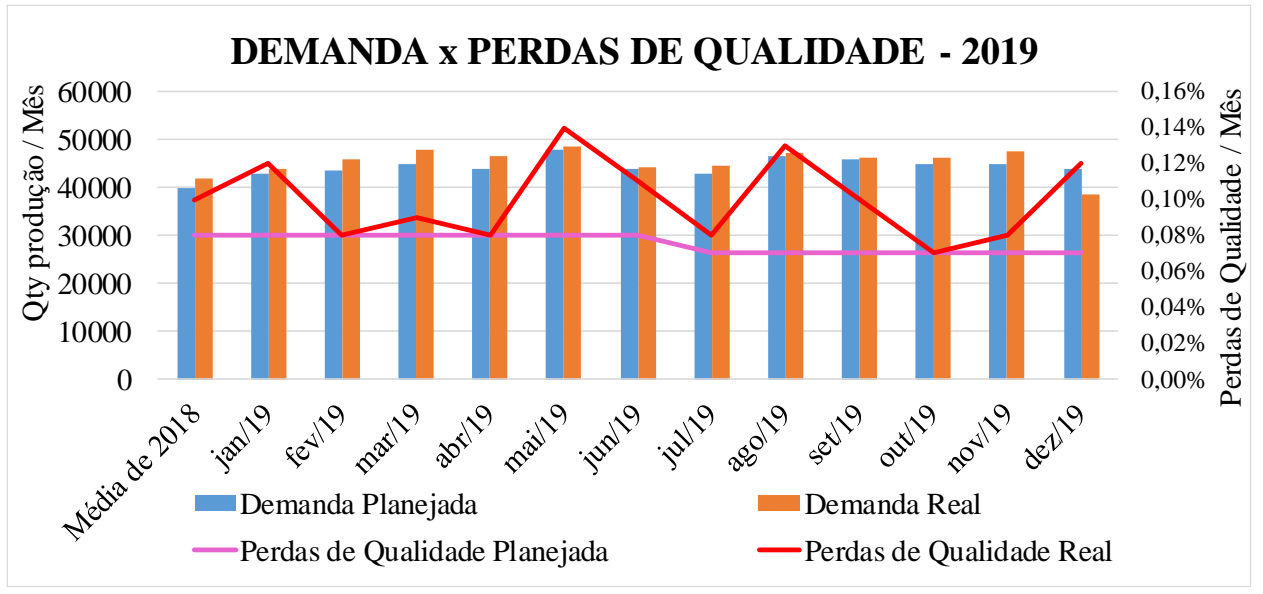

Fonte: Autores. 
$\mathrm{Na}$ Figura acima podemos identificar também que as perdas de qualidade real têm muitas oscilações e isso ocorre quando ações não estão sendo tomadas ou foram tomadas, porém não tem eficácia, ou seja, não atacou a causa raiz do problema ou ocorreu erro de planejamento.

Em relação a taxa de ocupação ou melhoria de produtividade, podemos afirmar que ocorrendo anormalidade similar a perdas de qualidade, onde podemos observar na Figura 8 em que em alguns meses a ocupação ficou acima de 100\%, ou seja, foi necessário a realização de hora extra para atendimento a demanda do cliente e isso certamente afeta negativamente o indicador de custo da organização

Figura 8 - Taxa de ocupação em 2019.

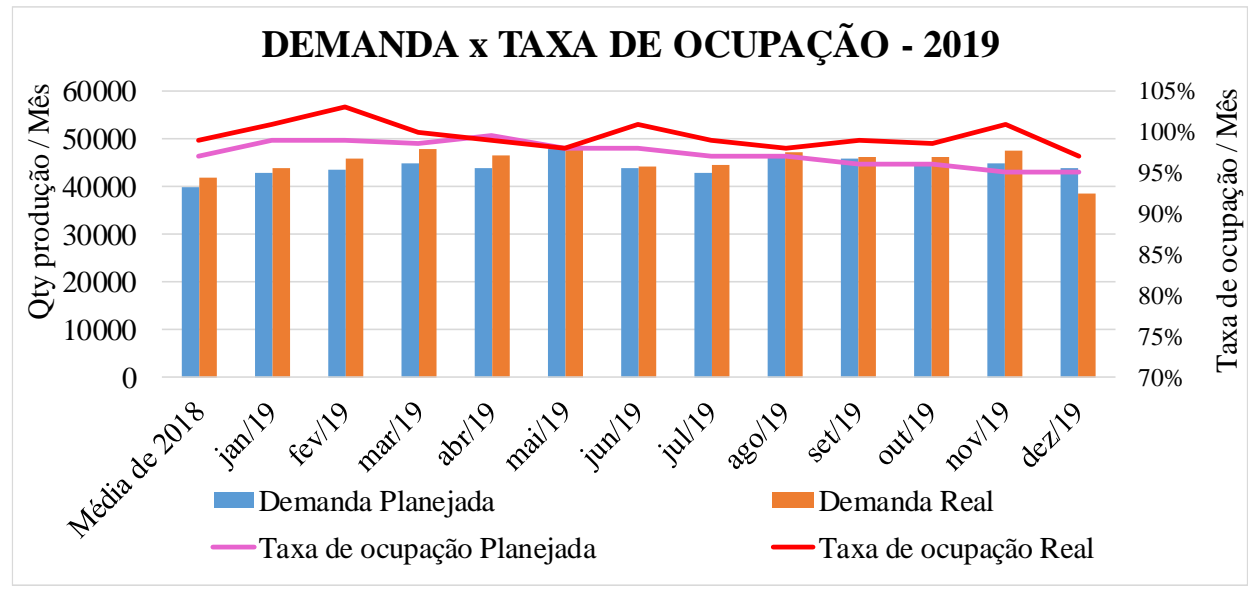

Fonte: Autores.

Todo o cenário exposto a cima é reflexo da não realização das atividades conforme o planejado e consequentemente os indicadores como perdas de qualidade e produtividade são impactados negativamente.

\subsection{Benefícios da etapa 3}

Com as informações de identificação do problema e o reconhecimento da situação, facilitou a definição das metas a serem alcançadas, pois já temos informações da dimensão do problema e consequentemente o quanto estamos distantes do esperado pela companhia. A contribuição principal desta etapa, foi que serviu como termômetro para o desenvolvimento das atividades.

\subsection{Benefícios da etapa 4}

Esta etapa nos mostrou como principal mérito o quanto é importante entender o mecanismo que gerou a falha ou alguma anormalidade na atividade. Sem realização de forma correta desta etapa, o plano de ação certamente não irá comtemplar a ações na causa raiz e consequentemente os objetivos traçados poderá não ser alcançado. Com objetivo de evitar que os resultados do ano de 2019 referente a qualidade e produtividade viessem a ocorrer em 2020, a alta direção da companhia realizou entrevistas com todos responsáveis de cada melhoria para extrair os principais problemas que prejudicaram o atingimento das metas no ano fiscal de 2019 e o resultado podemos observar na Tabela 2. 
Tabela 2 - Levantamento de problemas.

\begin{tabular}{|c|c|c|c|c|c|c|c|c|}
\hline \multirow{2}{*}{$\begin{array}{l}\text { DESCRIÇÃO DO } \\
\text { PROBLEMA } \\
\text { ENCONTRADO }\end{array}$} & \multicolumn{3}{|c|}{ MECANISMO DA FALHA } & \multicolumn{4}{|c|}{$\begin{array}{c}\text { FASE DO } \\
\text { PDCA }\end{array}$} & \multirow{2}{*}{$\begin{array}{c}\text { MOTIVOS PARA } \\
\text { DEFINIÇÃO DA FASE DO } \\
\text { PDCA }\end{array}$} \\
\hline & Gerenciamento & Sistemática & $\begin{array}{c}\text { Falta de } \\
\text { conhecimento }\end{array}$ & $\mathrm{P}$ & $\mathrm{D}$ & $\mathrm{C}$ & A & \\
\hline $\begin{array}{c}\text { Dimensionamento do } \\
\text { tempo para execução da } \\
\text { atividade - Tempo } \\
\text { Planejado menor que o } \\
\text { real }\end{array}$ & - & $X$ & $X$ & $\mathrm{X}$ & - & - & - & $\begin{array}{l}\text { Não fez estudo mais } \\
\text { aprofundado da atividade } \\
\text { durante o } \\
\text { reconhecimento da } \\
\text { situação atual }\end{array}$ \\
\hline $\begin{array}{c}\text { Durante a realização de } \\
\text { teste ocorreu um } \\
\text { problema que não foi } \\
\text { previsto }\end{array}$ & $X$ & $X$ & $X$ & $\mathrm{X}$ & $X$ & - & - & $\begin{array}{c}\text { Não fez análise dos } \\
\text { mecanismos de falhas } \\
\text { para posterior tomada de } \\
\text { ações }\end{array}$ \\
\hline $\begin{array}{l}\text { Resultado da atividade } \\
\text { não alcançou o objetivo }\end{array}$ & $X$ & $X$ & X & $X$ & - & - & - & $\begin{array}{l}\text { Na definição das metas } \\
\text { desconsiderou fatores } \\
\text { indiretos que poderiam } \\
\text { afetar na atividade }\end{array}$ \\
\hline \multirow[b]{2}{*}{$\begin{array}{c}\text { Faltou verificação e } \\
\text { direcionamento durante } \\
\text { o desenvolvimento da } \\
\text { atividade }\end{array}$} & \multirow[b]{2}{*}{$X$} & \multirow[b]{2}{*}{$X$} & \multirow[b]{2}{*}{-} & $\mathrm{X}$ & - & $\mathrm{X}$ & - & $\begin{array}{l}\text { O projeto não tinha a } \\
\text { metodologia de gestão a } \\
\text { vista }\end{array}$ \\
\hline & & & & $X$ & - & - & - & $\begin{array}{l}\text { Cronograma de execução } \\
\text { de atividades com } \\
\text { informações faltantes } \\
\text { como por exemplo } \\
\text { periodicidade de } \\
\text { verificação do andamento } \\
\text { da atividade para } \\
\text { possíveis direcionamento }\end{array}$ \\
\hline $\begin{array}{l}\text { Coleta de dados para } \\
\text { realização do projeto } \\
\text { insuficiente }\end{array}$ & - & X & $X$ & $\mathrm{X}$ & - & - & - & $\begin{array}{c}\text { Reconhecimento da } \\
\text { situação atual feita de } \\
\text { maneira incorreta }\end{array}$ \\
\hline $\begin{array}{c}\text { Priorização das } \\
\text { atividades de maneira } \\
\text { incorreta }\end{array}$ & $X$ & $X$ & $X$ & $\mathrm{X}$ & - & - & - & $\begin{array}{l}\text { Análise da causa raiz do } \\
\text { problema feita de } \\
\text { maneira incorreta }\end{array}$ \\
\hline $\begin{array}{l}\text { Viabilidade do projeto } \\
\text { não atende os requisitos } \\
\text { definido pela empresa }\end{array}$ & X & $X$ & $X$ & $X$ & - & - & - & $\begin{array}{c}\text { Reconhecimento da } \\
\text { situação atual e ideal } \\
\text { feita de maneira incorreta }\end{array}$ \\
\hline
\end{tabular}

Fonte: Empresa estudada.

A tabela acima nos mostra que grande parte das atividades planejadas não atingiram os seus objetivos devido a problemas na fase Planejamento (P) do ciclo PDCA. Para o atendimento dos objetivos da companhia, a alta direção determinou a criação de um grupo de estudo envolvendo todos os departamentos da empresa onde o objetivo foi reduzir ou até mesmo eliminar as anormalidades ocorridas no ano de 2019 e além disso a melhoria precisava abranger os setores operacionais e principalmente o gerenciamento de atividade da empresa.

\subsection{Benefícios da etapa 5}

A implementação do plano de ação com informações do problema, o por que, onde, prazo para tomada de ação e o como será feito o desenvolvimento, teve como principal benefício, permitir o monitoramento do andamento da atividade de forma eficiente e além disso, através deste monitoramento foi possível tomar ações de forma rápida quando o desenvolvimento da atividade não estava conforme o planejado. Devemos destacar que é preciso existir uma sistemática de verificação do andamento das atividades e o cronograma acima deve ficar visível a todos, pois desta forma facilita o gerenciamento da atividade ou suporte em possíveis dificuldades.

\subsection{Benefícios da etapa 6}

Como ponto importante e benéfico desta etapa podemos destacar o compartilhamento de informações diárias entre os envolvidos durante as reuniões e só foi possível porque a companhia adotou uma sistemática de checagem das atividades, onde 
diariamente é feita pelos gestores e uma vez por semana é feita a verificação pela diretoria. Outro ponto a ser destacado é que o andamento das atividades ficou visível a todos em um painel de gestão à vista.

\subsection{Benefícios da etapa 7}

Para facilitar a verificação da eficácia das ações implantadas, foi elaborado um fluxograma de avaliação de eficácia das ações, conforme apresentado na Figura 9 e o principal objetivo foi padronização da sistemática de verificação dos resultados e com isso proporcionou um direcionamento mais eficaz caso o resultado não seja satisfatório.

Figura 9 - Fluxograma de avaliação de eficácia das ações.



Fonte: Autores.

Nesta etapa também foi possível confirmar todos os méritos das etapas anteriores e além disso, verificar se as metas planejadas foram alcançadas. Para o estudo de caso em questão foi planejado no ano de 2020 a redução de perdas de qualidade em $0,01 \%$ e redução da taxa de ocupação em $0,16 \%$, ambos indicadores tiveram como referência o ano de 2019 e os resultados obtidos, serão mostrados nas Figuras 10 e 11 respectivamente. O ciclo PDCA começou a ser utilizada para implantações de melhorias na organização no mês de maio de 2020 e os primeiros méritos começaram a aparecer em aproximadamente um mês após início da utilização. 
Figura 10 - Perdas de qualidade em 2020.

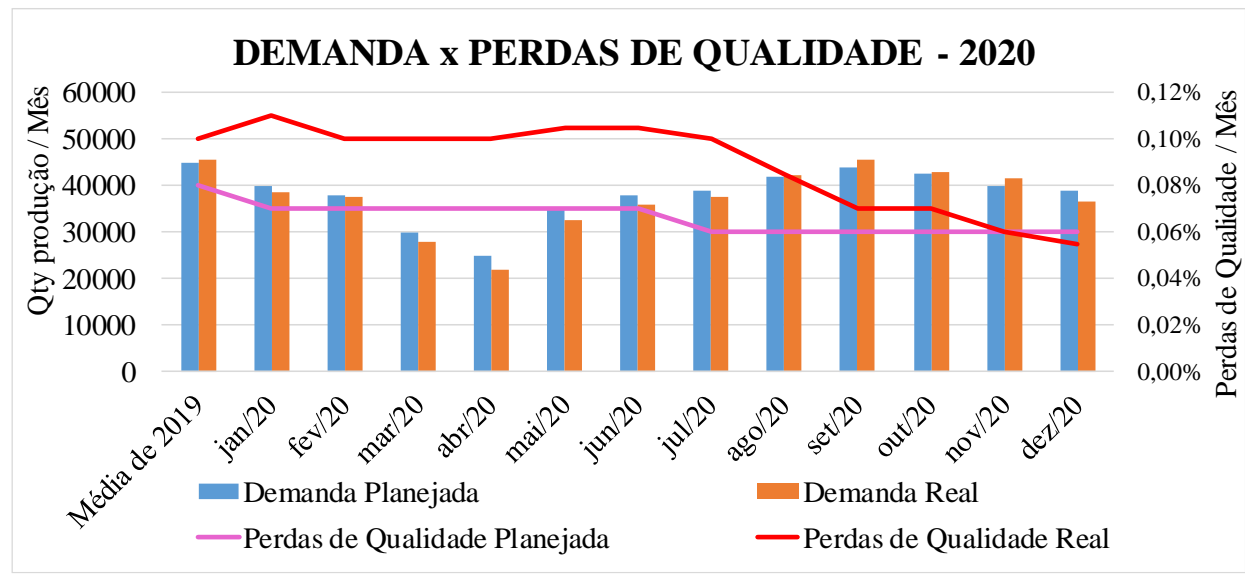

Fonte: Autores.

Pelos resultados encontrados na figura acima, é possível verificar que a partir de maio o comportamento da curva de perdas de qualidade real começou a apresentar tendência de melhorias e além disso, começamos a ter previsibilidade nos resultados dos meses seguintes, visto que a curva não apresenta oscilações significantes e esse comportamento ratifica que não estar ocorrendo reincidência de anormalidade nas melhorias realizadas. Outro fator importante que devemos salientar é que apesar de a maioria dos meses a meta não ser atingida, nos últimos dois meses foi possível atender e a tendência é que em 2021 os objetivos sejam alcançados em todos os meses.

Com relação a taxa de ocupação, mesmo não atingindo a meta definida é possível verificar que houve melhoria ao longo de 2020.

Figura 11 - Taxa de ocupação em 2020.



Fonte: Autores.

Da mesma forma que foi verificado no indicador de perdas de qualidade, o indicador de taxa de ocupação real também reduziu as oscilações e com isso passamos a ter previsibilidade, facilitando as priorizações para tomada de ações.

Os resultados de perdas de qualidade e taxa de ocupação alcançados em 2020, foram satisfatórios quando comparado com o ano de 2019, isso mostra um avanço no desenvolvimento das atividades e execução de melhorias na companhia e estes resultados refletem positivamente nos indicadores. 


\subsection{Benefícios da etapa 8}

Esta etapa nos permitiu refletir sobre a caminho a ser seguido, como fazer a divulgação dos conhecimentos adquiridos e o que fazer com eventuais problemas remanescente e além disso, esta fase nos propiciou a continuidade do ciclo PDCA através padronização das atividades que tiveram êxito e atualizações ou criação de documentações do processo, desta forma foi possível iniciar a abrangência lateral para outras linhas de produção e produtos, referente ao uso do ciclo PDCA.

\section{Considerações Finais}

Neste estudo foi visto que através da utilização do ciclo PDCA, se teve um conhecimento da situação da empresa antes da utilização da metodologia, onde foi identificado os principais problemas. Em relação aos resultados das metas, a meta referente a perdas de qualidade para ano de 2020, considerando os últimos meses foi atingida, que era redução de $0,01 \%$, enquanto que a taxa de ocupação, houve redução de $0,13 \%$, não atingindo a meta que era de $0,16 \%$, porém levando em consideração os resultados dos últimos meses de 2020, podemos afirmar que a meta será atingida nos primeiros meses de 2021.

Outro ponto relevante que podemos destacar é os benefícios para a empresa, pois com a utilização do ciclo PDCA, foi estabelecido um compromisso com a melhoria constante, o que permite que a empresa permaneça competitiva e constantemente melhore e se adapte. Outro fator relevante é que os processos se tornaram melhores e mais bem compreendidos e além disso a ferramenta apresenta uma estrutura fácil de se entender e consequentemente facilitando a aplicação da mesma.

Finalmente, conclui-se que, através da utilização da metodologia PDCA, resultados relevantes foram alcançados para esta empresa do Polo Industrial de Manaus analisada e certamente a decisão de implementar esta metodologia para toda a empresa, provavelmente não terá como restrição a dificuldade de compreendê-la, mas sim o empenho dispendido na sua aplicação

Em relação a limitações do presente trabalho, podemos destacar o espaço amostral utilizado para aplicação inicial da melhoria, que corresponde a aproximadamente $12 \%$ da organização. Certamente um espaço amostral maior deixaria ainda mais confiável a nova forma de utilização da metodologia.

Como sugestão futura, temos a ideia de fazer abrangência e aplicação deste estudo para todos os departamentos da empresa e expandir esta lógica para outras unidades a nível nacional e mundial, e além disso para comunidade em geral;

\section{Referências}

Asti, Vera, A. (1979) Metodologia da pesquisa científica. (5a ed.), Globo.

Badiru, A. B. J. (1993) Practitioner's guide to quality and process improvement. Chaman \& hall.

Campos, V. F. (1996) Gerenciamento pelas diretrizes. Fundação Christiano Ottoni Escola de Engenharia da UFMG.

Campos, V, F. (2001) Gerenciamento da rotina de trabalho do dia-dia. Editora de desenvolvimento Gerencial.

Chiavenato, I. (1999) Teoria geral da administração (6a ed.), Campus, V 1.

Clark, A. B. (2001) How managers can use the Shewhart PDCA Cycle to get better results. Houston: Jesse H. Jones Scholl of Business - Texas Southern University.

Correa, C. V. F. (2017) O que importa é resultado. Sextante, 2017.

De Paula, J. (2021) Utilização do ciclo PDCA e aplicação do Milk run em um processo de logística reversa em uma indústria de alimentos: um estudo de caso. UFES. Brazilian Journal of Production Engineering, 7(2), 16-30.

Duppre, T. C. (2015) Aplicação do ciclo PDCA visando a redução dos índices de refugo de peças: pesquisa-ação em uma empresa do setor de autopeças. Encontro Nacional de Engenharia de Produção (ENEGEP), Anais do ENEGEP.

Estrela, C. (2018) Metodologia Científica: Ciência, Ensino, Pesquisa. Editora Artes Médicas.

Gerolamo, M. C. (2014) Quality Management: How do Brazilian Companies use it? Procedia - Social and Behavioral Sciences, 143,995 - 1000. 
Iwahana, A. (2013) The art of producing using PDCA cycle.

Jindsong, L. Xiaoguang Z. Tianshu, Z. \& Ren. M. (2010) Medical Process Management by Applying PDCA to EMR.

Kitahara, S. (2016) PDCA philosophy for manufacturing companies.

Marshall, Junior, I. et al. (2010) Gestão da qualidade. (10a ed.), Editora FGV.

Melo, C. P. \& Caramori, E. J. (2001) PDCA Método de melhorias para empresas de manufatura - versão 2.0. Fundação de desenvolvimento Gerencial.

Moen, R. D. \& Norman, C. L. (2006) Evolution of the PDCA Cycle.

Moura, L. R. (1997) Qualidade simplesmente total: uma abordagem simples e prática da gestão da qualidade. Qualitymark Ed.

Peleteiro, F. S. (2018) Estudo sobre ganho de qualidade e produtividade mediante a aplicação do PDCA. - Universidade Federal do Rio de Janeiro, Rio de Janeiro.

PDCA Cycle. http://www.hci.com.au/heisite2/toolkit/pdcacyel.htm.

Pereira A. S. et al. (2018) Metodologia da pesquisa científica. UFSM.

Pereira, F. (2021) Aplicabilidade da ferramenta PDCA no transporte logístico fluvial de hortifrúti tomate: um estudo de caso. UFPA. Brazilian Journal of Development, 7(3), 27946-27957.

Rodrigues, A. (2017) A Utilização do ciclo PDCA para melhoria da qualidade na manutenção de SHUTS: um estudo de caso, UFSC. 9(18), 48-70.

Sousa, R. (2020) Aplicação do Ciclo PDCA para redução de Custos e Perdas em uma Distribuidora de Hortifruti: um estudo de caso. UFPE. Journal of Perspectives in Management-JPM, 4, 68-83.

Souza, R. (1997) Metodologia para desenvolvimento e implantação de sistemas de gestão de qualidade em empresas construtoras de pequeno e médio porte. 1997, 387p. Tese - Escola Politécnica, Universidade de São Paulo.

Suski, C. (2020) Redução de custos de insertos no processo de usinagem por meio da metodologia PDCA: Um estudo de caso. IFSC, Revista de Tecnologia Aplicada, 9(3), 33-44.

Suzuki, M. (2000) Implementation of Project management based os QES and those issues in japanese construction industry and in Kumagaigumi. International Conference On Implemetation Of Costruction Quality And Relates Systems, Lisboa.

Taylor, M. J. McNicolas, C., Nicolay, C. Darzi, A. Bell, D., \& Reed, J. E. (2013). Systematic review of the application of the plan-do-study-act method to improve quality in healthcare. BMJ quality \& safety 0, 1-9.

The PDCA Cycle. The clinician's black bag of quality imporvement tools. http://www.dartmouth.edu/ ocer/CQI/PDCA.html.

Yin, R. K. (2015) O Estudo de caso. Bookman. 